\title{
The case for open source appropriate technology
}

\author{
Joshua M. Pearce
}

Received: 27 September 2011/ Accepted: 5 January 2012/Published online: 19 January 2012

(C) The Author(s) 2012. This article is published with open access at Springerlink.com

\begin{abstract}
Much of the widespread poverty, environmental desecration, and waste of human life seen around the globe could be prevented by known (to humanity as a whole) technologies, many of which are simply not available to those that need it. This lack of access to critical information for sustainable development is directly responsible for a morally and ethically unacceptable level of human suffering and death. A solution to this general problem is the concept of open source appropriate technology or OSAT, which refers to technologies that provide for sustainable development while being designed in the same fashion as free and open source software. OSAT is made up of technologies that are easily and economically utilized from readily available resources by local communities to meet their needs and must meet the boundary conditions set by environmental, cultural, economic, and educational resource constraints of the local community. This paper explores both the open source and appropriate technology aspects of OSAT to create a paradigm, in which anyone can both learn how to make and use needed technologies free of intellectual property concerns. At the same time, anyone can also add to the collective open source knowledge ecosystem or knowledge commons by contributing ideas, designs, observations, experimental data, deployment logs, etc. It is argued that if OSAT continues to grow and takes hold globally creating a vibrant virtual community to share technology plans and experiences, a new technological revolution built on a dispersed network of innovators working together to create a just sustainable world is possible.
\end{abstract}

Keywords Appropriate technology $\cdot$ Knowledge commons · Open access · Open design · Open source $\cdot$ Sustainable development

Readers should send their comments on this article to BhaskarNath@aol.com within 3 months of publication of this issue.

J. M. Pearce $(\bowtie)$

Department of Materials Science \& Engineering and the Department of Electrical \& Computer Engineering, Michigan Technological University, 601 M\&M Building, 1400 Townsend Drive, Houghton, MI 49931-1295, USA

e-mail: pearce@mtu.edu 


\section{Introduction}

Even a superficial review of global environmental conditions results in a rather bleak outlook for the sustainability of the world's major ecological systems. Simultaneously, billions are mired in poverty, and even those in the developed rich countries find their economic situations uncomfortably precarious. The optimists' position is that accelerated progress in technology will rescue global society even from global-scale problems like climate destabilization. Support for this position is legion. It is undeniable that technological development has provided great benefits to humankind in medicine and many other fields, and technology is indeed developing faster now than ever before. There are more $\mathrm{PhDs}$, scientists, and engineers working now than the world has ever seen. More papers are being written and new scientific journals are proliferating at an astounding rate. ${ }^{1}$ Unfortunately, the vast majority of this research, and the knowledge created, is not focused on problems related to sustainable development and surprisingly, even much of it, that is, is effectively removed from deployment by intellectual property law (e.g. patents, copyrights, and trademarks) (Pearce and Mushtaq 2009). The results of this restricted and closed model of technological development are the widespread poverty and environmental desecration seen around the globe, which is directly responsible for a morally and ethically unacceptable level of human suffering and death. For example, more than 10 million children under the age of five die each year from preventable causes (WHO 2007). This waste of human life could be prevented by known (to humanity as a whole) technologies, many of which are simply not available to those that need it. Availability is restricted by both the cost of access (such as pay-to-view articles on renewable electricity generation under copyright by the IEEE) ${ }^{2}$ and by companies wielding patent law to maximize profit at the cost of human lives (e.g. restricting the sale of antiretroviral drugs to treat HIV in Africa) (Shantharam 2005). A solution to this general problem of access to critical information for sustainable development is the growth of open source appropriate technology.

\section{What is open source appropriate technology?}

Open source appropriate technology (OSAT) refers to technologies that provide for sustainable development while being designed in the same fashion as free and open source software (Buitenhuis, et al. 2010). The open software movement has produced a community of hackers and computer programmers whose shared goal is to work together to develop better computer software (DiBona et al. 1999). If open source software such as GNU/Linux is compared against Microsoft's centralized and closed system of software development, a surprising result surfaces. A neutral technical assessment finds that open source software, developed mostly by unpaid volunteers, is often of superior quality to the software developed by one of the most powerful companies in the history of the world employing unquestionably extremely intelligent people (Bonaccorsi and Rossi 2003). This remarkable result stands against conventional wisdom that would argue the profit motive,

\footnotetext{
${ }^{1}$ The rate of growth of scholars has been about 3\%/year, scholarly journals is 3.25\%/year, and articles published in them grow about 3.5\%/year (Mabe 2003; Ware and Mabe 2009). For more information, see recent discussion on the future of scholarly publishing particularly as it relates to open access by Björk, Roos and Lauri (2008), Phillips (2010) and Tenopir and King (2009).

${ }^{2}$ IEEE articles are now available online at http://ieeexplore.ieee.org/Xplore/guesthome.jsp.

However, even conference proceedings such as the Pearce and Mushtaq (2009) article on OSAT costs US\$30 for non-subscribers.
} 
and market forces would enable Microsoft to develop superior software to any random group of volunteers. Microsoft is a large company, with annual revenue of over $\$ 40$ billion, yet many of its products suffer from technical drawbacks that include bloat, lack of reliability, and security holes. Microsoft remains dominant largely because of inertia, but Linux eats up an ever larger market share (particularly in servers), because open source is simply more efficient and adaptable than closed, hierarchical systems (Kogut and Metiu 2001). This is due, historically at least, in a large part because a lot more people collaborate on Linux than on Microsoft products. Where Microsoft might utilize a few thousand programmers and software engineers to debug their code, the Linux community has access to hundreds of thousands of programmers debugging, rewriting, and submitting code. ${ }^{3}$ This type of mass-scale collaboration is driving the success of Web 2.0 applications that emphasize online collaboration and sharing among users (examples include social networking sites and wikis). As the Aggroblogger asked several years ago (Aragona 2005): "Now that Open Source has come of age, the question is not: Is it better than closed software? But rather: To what other systems, outside of software, can we apply the concepts of Open Source and public ownership?"

One area of technology ripe for the development of open source is appropriate technologies (AT), which was first popularized by Schumacher (1973) in his classic book "Small is Beautiful: A Study of Economics As If People Mattered." Appropriate technology can be defined as those technologies that are easily and economically utilized from readily available resources by local communities to meet their needs. Although much OSAT is made up of relatively simple and non-complex technologies (e.g. rope pumps), OSAT also encompasses complex and state-of-art devices such as those developed with nanotechnology (Mushtaq and Pearce 2012). The key of AT is that it must meet the boundary conditions set by environmental, cultural, economic, and educational resource constraints of the local community (Buitenhuis et al. 2010; Pearce 2009). There are numerous groups, non-profit organizations, universities, companies and individuals that have embraced the open source paradigm when working on AT (Pearce et al. 2008). ${ }^{4}$

\section{OSAT in action}

The Appropedia foundation perhaps offers one of the best examples of OSAT in action as it harnesses the power of distributed peer review and transparency of process. The Appropedia foundation hosts www.appropedia.org the primary site for collaborative solutions in sustainability, poverty reduction, and international development on the internet. Appropedia is an advertising-free Mediawiki-based Web site like Wikipedia, a Web site where a large number of participants (including you) are allowed to create and modify the content directly from their Web browsers. The use of such OSAT Web sites has enormous potential to assist in sustainable development because it simplifies the administration of collaboratively organizing information, project examples, best practices, and

\footnotetext{
${ }^{3}$ In fact, even Microsoft is now embracing some components of open source development. A Microsoft representative has recently stated that both SQL Server and the Windows Azure teams are committed to the Hadoop open source platform for the long term (Metz 2011).

4 For examples, see (Zelenika and Pearce 2011) and consider the rapidly growing body of OSAT work done by groups that publish OSAT and encourage open collaboration including Appropriate Technology Collaborative (ATC), Appropriate Infrastructure Development Group (AIDG), Ayzh, Catalytic Communities (CatCom), Compatible Technology International (CTI), Digital Green, International Development Research Center (IDRC), Kopernik, and practical action.
} 
"how tos" (Pearce et al. 2010). OSAT on the Internet is set to expand rapidly as other organizations utilize its information transfer and collaboration capabilities (Buitenhuis et al. 2010; Zelinki-Zovko and Pearce 2011). Following the OSAT paradigm, anyone can both learn how to make and use AT free of IP concerns. At the same time, anyone can also add to the collective open source knowledge ecosystem or "knowledge commons" by contributing ideas, designs, observations, experimental data, deployment logs, etc. The built-in continuous peer review should result in better quality, higher reliability, and more flexibility than conventional design/patenting of technologies. ${ }^{5}$ The free nature of the knowledge also provides lower costs, particularly for those technologies that do not benefit to a large degree from scale of manufacture. Already the open source paradigm is gaining momentum in hardware, with such devices as the open source 3D printers for both the design, but also the production of OSAT (Pearce et al. 2010). In this case, open source electronic prototyping platforms (Arduino) can be used to build a self-replicating 3D printer (RepRap), capable of manufacturing solid complex pieces without the need for expensive industrial infrastructure. RepRap's software and the printer designs are open and available free of charge, while the printer can also make most of its own components. ${ }^{6}$ In addition to large complex prints such as its own parts, the RepRap is also capable of making small simple components of OSAT. For example, consider the DremelFuge chuck, which is a printable rotor for centrifuging standard microcentrifuge tubes and miniprep columns (Garvey 2009). The DremelFuge can be used in the field as an extremely inexpensive centrifuge (costing under $\$ 50$ - primarily the cost of the drill-compared to commercial systems, which costs thousands of dollars). It can be used for any application in development needing a microcentrifuge including medical, biochemistry, or education in the sciences. Thus, OSAT also enables a path to the end to predatory intellectual property lock-in. This is particularly important in the context of technology focused on relieving suffering and saving lives in the developing world.

Conventional wisdom again would predict that any type of intellectual property development created using open source paradigms should not be able to compete with the highly successful and well-demonstrated economic-free market. Surprisingly, the counter argument comes from the development of science and engineering that underpins the technologies that make up some of the free market's greatest success stories. Although the vast majority of scientists and engineers are employed by the free market including those at academic institutions, the science and engineering we do at academic institutions is generally published for everyone else in their particular field to see. This open source science is currently being expanded to the rest of the world's population as the debate in academia is shifting to favor open access journals or open access options for manuscripts

\footnotetext{
5 It should be noted that an open source approach is not necessarily an instantaneous panacea for design of anything. Although, for example, there are many excellent open source software packages, there are also a notable number of poorly coded and low performance open source programs. Thus, there is no implied guarantee (or even great likelihood) that any one specific piece of OSAT will be of high quality, but rather the open source process tends to create gems alongside many unfinished or less functional concept and products. These underdeveloped concepts can all be built on by others and improved for the future.

6 The RepRap project not only is an open hardware project, but it uses open source software and wiki technology to spread the results in the development community. See http://www.reprap.org Work is also underway to utilize waste plastic in an open source hardware project called the Recyclebot (http://www.thingiverse.com/thing:12948) to provide the feedstock filament for the RepRaps. The vision for this work is to enable anyone to literally print themselves out of poverty by utilizing a combination of Linux, open source designs on the Internet, Recyclebot, and RepRap technologies. This technical work is in its infancy, but the potential is clear to a growing number of hackers and researchers assisting on the collaborative projects.
} 
(e.g. Springer's Open Choice for Environment, Development and Sustainability), which are available to any reader in the world free of charge. ${ }^{7}$ The primary advantage of open access is that the content is available to users everywhere regardless of affiliation with a subscribing library. This benefits

- authors, as they like to see their papers cited and read more often;

- the development of science and engineering as it enables academic readers at institutions that cannot afford the journal (as in many developing nations) to participate; and

- the general public as they can more easily see how research effects their lives (e.g. patients can keep up with the latest medical research).

Copyright, patents, and trademarks are often lumped together under the term of intellectual property (IP). However, if one uses information, it does not prevent someone else from using it, and in fact if both people use it, it may actually accelerate the development of additional information or innovation. Because of this exclusive monopoly rights governing IP, information is locked up and often prohibits or slows innovation, which is the exact opposite of the intended purpose of modern IP laws (Kogut and Metiu 2001). IP laws rewarding innovators for inventions or art/writing are meant to encourage innovation. In some industries, this aim may be achieved with patents, and in others, this is highly questionable such as in software (Merges and Nelson 1990). In the case of AT, there is an unavoidable moral and ethical dilemma. Is it acceptable to withhold information that could save the world's poorest people from suffering and death? For most individual and academic researchers, the answer is obvious.

For companies working in the AT field, the answer is more opaque. Although developing world problems are portioned remarkably low support for solutions (as compared to junk food, gaming, makeup, or most any other categories of consumer products), many researchers, companies, and academics do work on products meant to assist sustainable development. Companies that do develop and profit from the sale of AT often can deliver affordable life affirming products and services to the "bottom of the pyramid" using conventional mass-scale manufacturing and business practices. How can they remain economically sustainable while opening their IP? One potential solution to this question comes from an unlikely source: Mrs. T's Pierogies. This is a small Pennsylvanian company that has a very successful business of selling pierogies (mashed potato filled pasta dumplings) — they ship 11.4 million a week (Pearce and Mushtaq 2009). Recently, they volunteered all of the IP for the making of pierogies in a documentary: walked the viewer through their plant and then showed step by step how to make a pierogie at home from scratch. At the end of the documentary, they pointed out that although they showed you exactly how to make it, making pierogies is hard work and many people do not have the time... in that case consider buying Mrs. T's! A similar model ${ }^{8}$ is being used by dozens of successful open source hardware companies and could be followed for OSAT, so the

\footnotetext{
7 For more information on Open Choice see: http://www.springer.com/open+access/open+choice?SGWID=040359-0-0-0 and for the Directory of Open access Journals see: http://www.doaj.org/.

8 There are numerous examples of hardware companies with over a million in revenue, whose main products are open source including Adafruit Industries, Arduino, BeagleBoard, Buglabs, Chumby, Dangerous Prototypes, DIY Drones, Evil Mad Scientist Labs, Liquidware, Makerbot Industries, Maker Shed, Parallax, Seed Studios, Solarbotics, and Sparkfun Electronics. Phillip Torrone and Limor Fried from Adafruit Industries predict the open source hardware industry will break \$1 billion in revenue by 2015 as it expands rapidly (Torrone and Fried 2010). For more information on potential business models for OSAT development, see Buitenhuis et al. (2010).
} 
information necessary to provide the basic needs of the world's poorest could be open sourced.

\section{A sustainable future}

Throughout the world, there exist research institutes, community groups, and non-governmental organizations working with different technological innovations to alleviate poverty and mitigate the destruction caused by excesses of consumer culture. For the most part, they remain disconnected, often re-inventing the proverbial wheel again and again although their counterparts in another part of the world may have already designed and debugged a similar technology. Clearly, appropriate technology development could benefit greatly from the application of an open source model. OSAT could fall within the legal framework of an AT General Public License (GPL), where those plans can be used freely, modified, and republished under the same AT GPL for those in the future all over the world to benefit from. ${ }^{9}$

Consider the effect of open source appropriate technology taking hold creating a vibrant virtual community to share OSAT plans and experiences. OSAT venues like Appropedia are enabling designers and field workers to download plans of water pumps, wind mills, basic medicines, passive solar, and many other appropriate technologies. In this way, open source appropriate technology will become a true rival to the paradigms of the development of technology that have dominated civilization since the industrial revolution. A new revolution built on a dispersed network of innovators, inventors, and researchers working together to create a just sustainable world will be created. Join us.

Open Access This article is distributed under the terms of the Creative Commons Attribution Noncommercial License which permits any noncommercial use, distribution, and reproduction in any medium, provided the original author(s) and source are credited.

\section{References}

Aragona, F. (2005). Open sourcing appropriate technology part I. Agricultural Innovations. (Nov 17, 2005). http://agroinnovations.com/blog/2005/11/17/open-sourcing-appropriate-technology-part-i/. Accessed Dec 192011.

Björk, B., Roos, A. \& Lauri, M. (2008). Global annual volume of peer reviewed scholarly articles and the share available via different Open Access options. In: Proceedings of the ELPUB2008 Conference on Electronic Publishing, Toronto, Canada, June 2008. http://oacs.shh.fi/publications/elpub-2008.pdf. Accessed Dec 192011.

Bonaccorsi, A., \& Rossi, C. (2003). Why open source software can succeed. Research Policy, 32, $1243-1258$.

\footnotetext{
9 It should be noted that this licensing challenge can be significantly more complex and is an area of needed future work. Whereas copyright is automatic, patents must not only be applied for, but also entail a considerable capital investment. This investment is not only to obtain the legal protection of the patent, but also to effectively defend the patent in court. Without this defense, the patent is useless. Thus, open sourcing patentable ideas faces unique challenges. In theory, by creating "open access" to ideas, future potential patents lose their viability as one would think the claim to novelty is eradicated. However, by looking at the current patent literature, it is clear that often trivial variations on well-understood technologies are able to gain patents and thus the only real value in patents is in the capacity to defend an idea in court. It is not clear that the current patent system would thus be effective enough to protect open sourced ideas from IP poaching by well funded individuals or companies.
} 
Buitenhuis, A. J., Zelenika, I., \& Pearce, J. M. (2010). Open design-based strategies to enhance appropriate technology development. In: Proceedings of the 14th Annual National Collegiate Inventors and Innovators Alliance Conference: Open, 1-12.

DiBona, C., Ockman, S., \& Stone, M. (1999). Open sources: Voices from the open source revolution. Sebastopol: O'Reilly \& Associates.

Garvey, C. (2009). DremelFuge-A One-piece centrifuge for rotary tools. Thingiverse 1483, http://www. thingiverse.com/thing:1483. Accessed Dec 192011.

Kogut, B., \& Metiu, A. (2001). Open-source software development and distributed innovation. Oxford Review of Economic Policy, 17(2), 248-264.

Merges, R. P., \& Nelson, R. R. (1990). On the complex economics of patent scope. Columbia Law Review, 90(4), 839-916.

Metz, C. (2011). Microsoft embraces elephant of open source. Wired. http://www.wired.com/wiredenter prise/2011/10/microsoft-and-hadoop/. Accessed Dec 192011.

Mushtaq, U., \& Pearce, J. M. (2012). Open source appropriate nanotechnology. In D. Maclurcan \& N. Radywyl (Eds.), Nanotechnology and global sustainability (pp. 191-213). Boca Raton: CRC Press.

Pearce, J. M. (2009). Appropedia as a tool for service learning in sustainable development. Journal of Education for Sustainable Development, 3(1), 47-55.

Pearce, J. M., Grafman, L., Colledge, T. \& Legg, R. (2008). Leveraging information technology, social entrepreneurship and global collaboration for just sustainable development. In: Proceedings of the 12th Annual National Collegiate Inventors and Innovators Alliance Conference, 201-210.

Pearce, J. M., Morris Blair, C., Laciak, K. J., Andrews, R., Nosrat, A., \& Zelenika-Zovko, I. (2010). 3-D printing of open source appropriate technologies for self-directed sustainable development. Journal of Sustainable Development, 3(4), 17-29.

Pearce, J. M. \& Mushtaq, U. (2009). Overcoming technical constraints for obtaining sustainable development with open source appropriate technology. Science and technology for humanity (TIC-STH), 2009 IEEE Toronto International Conference, 814-820.

Phillips, A. (2010). Blog to the future? journals publishing in the twenty-first century. Journal of Scholarly Publishing, 42(1), 16-30.

Schumacher, E. F. (1973). Small is beautiful: Economics as if people mattered. New York: Harper \& Row.

Shantharam, Y. (2005). The cost of life: patent laws, the WTO, and the HIV/AIDS pandemic. Undercurrent, 2(2), 48-56.

Tenopir, C. W., \& King, D. W. (2009). The growth of journals publishing. In B. Cope \& A. Phillips (Eds.), The future of the academic journal (pp. 105-124). Oxford: Chandos Publishing/Woodhead Publishing Ltd.

Torrone, P. \& Fried, L. (2010). Million dollar baby: Businesses designing and selling open source hardware, making millions. Presented at Foo Camp East 2010. http://vimeo.com/11407341. Accessed Dec 19 2011.

Ware, M. \& Mabe, M. (2009). The STM Report (Oxford: International Association of Scientific, Technical and Medical Publishers).http://www.stm-assoc.org/2009_10_13_MWC_STM_Report.pdf. Accessed Dec 192011.

World Health Organization (WHO). (2007). Facts and figures: Mortality report. http://www.wpro.who.int/ media_centre/fact_sheets/fs_20070801.htm. Accessed Dec 192011.

Zelenika-Zovko \& Pearce, J. M. (2011). Examining social barriers to open source appropriate technology and innovation through collaboration with Information and Communication Technology. 17th Annual International Sustainable Development Research Conference, 507-508.

Zelenika, I., \& Pearce, J. M. (2011). Barriers to appropriate technology growth in sustainable development. Journal of Sustainable Development, 4(6), 12-22. 